\title{
Commentary
}

\section{The Development of Cloud Computing Service Pricing-Based on the Life Cycle Theory}

\author{
Ruijie Zhao', Yu Zhao' \\ ${ }^{1}$ School of Business Administration, South China University of Technology, Guangzhou, China \\ ${ }^{2}$ School of Civil Engineering, Zhengzhou University, Zhengzhou, China
}

Email address:

bangbangtangzhile@163.com (Ruijie Zhao)

\section{To cite this article:}

Ruijie Zhao, Yu Zhao. The Development of Cloud Computing Service Pricing — Based on the Life Cycle Theory. Science Journal of Business and Management. Vol. 4, No. 3, 2016, pp. 102-107. doi: 10.11648/j.sjbm.20160403.16

Received: May 18, 2016; Accepted: May 28, 2016; Published: June 13, 2016

\begin{abstract}
The application of cloud computing is conducive to the efficient allocation of resources and cost reduction. With the vigorous development of cloud technology, more and more enterprises have involved in cloud services, so the competition among providers is increasingly fierce. How to formulate a reasonable price strategy is one of the current matters urgently needing solved for the long term benefit of the market. Only by establishing the good pricing strategy can enterprises better seize market and build a stable customer relationship. Based on the concept of cloud computing, this paper makes a brief comment on the study of the literature of the three types of pricing mechanisms in the business life cycle of cloud computing. It is pointed out that dynamic pricing can better meet the interests of both sides of supply and demand and become the main trend of future pricing. Future research needs to study the characteristics of the cloud service life cycle from the cloud services industry. Consider the long-term benefits of customers and suppliers, and develop dynamic pricing mechanism.
\end{abstract}

Keywords: Cloud Computing Services, Pricing, The Life Cycle

\section{Introduction}

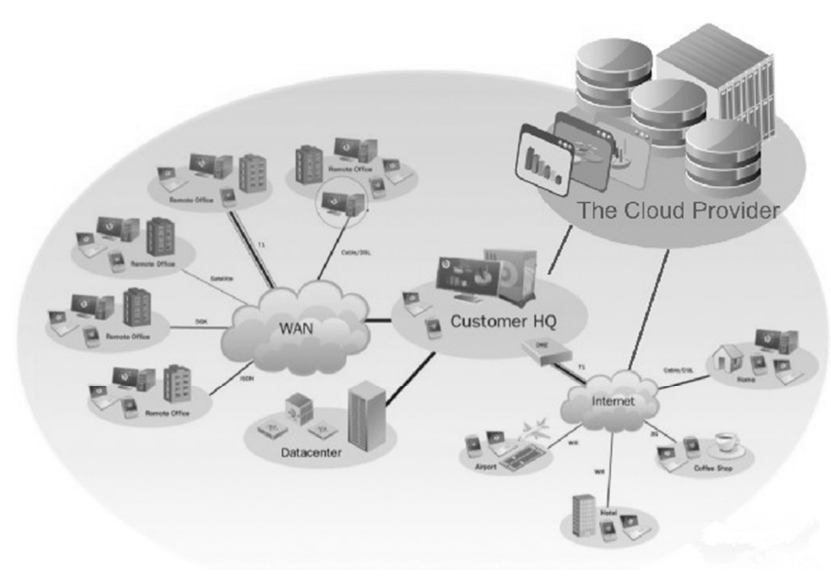

Figure 1. Cloud computing

Cloud computing services ("CCS") are changing the traditional network information services. The traditional way of network information service is completely based on the server as the center, connected to the computer processor through the cable support, and parasitic on the server or client. However, with the rapid development of globalization and the increasing amount of data, people on the network information service requirements are also constantly improved. Just as figure $1 \mathrm{CCS}$ is changing the traditional service model from direct to virtual services. It does not need to be added to the machine equipment to achieve the expansion of services to meet the needs of flexible. CCS users do not need to build their own information architecture (network, CPU, storage, Computing), platforms, software etc. Only need to pay a small amount of rent to get the right to use. CCS with the characteristics of "three easy" is reforming our times. "Three easy" refers to the CCS is easy to learn; simple operation, easy to be mastered; and low cost, easy to achieve low input and efficient use. According to the survey, 2015-2018 years, the global cloud services market will grow by an annual average of $26 \%$, while the China will close to $45 \%$ compound annual growth rate [1]. As shown in figure 2 , It is clear that the future 
of Chinese CCS market has an attractive market gaps. Iaas, SaaS and PaaS are three kinds of CCS delivery mode. In the face of such a broad prospect of cloud computing, the major companies have increased investment to seize the market. Since then, cloud computing has become a new round of global competition in the high ground.

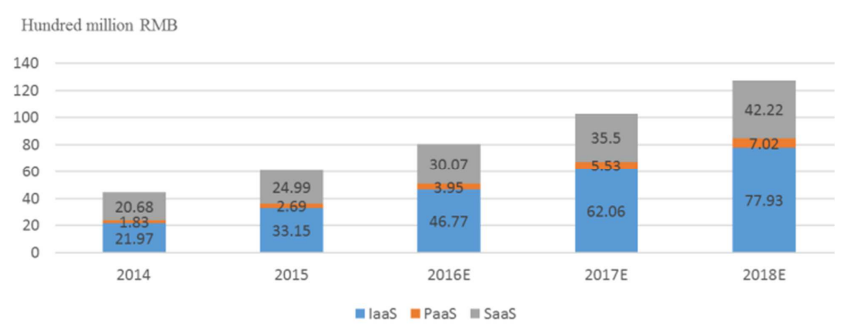

Figure 2. Public cloud market size forecast in China.

With the popularity of CCS, the issue of CCS business model has become increasingly prominent. Cloud computing has the characteristic that the marginal cost of the total cost is almost zero, so the traditional form of the traditional marginal cost pricing has been applied to the pricing of the model. In March 2014, Ali took the lead down the price of cloud computing services, followed by Amazon, Microsoft, Google, and other giants have greatly reduced prices. In the wave of price cuts, how to protect the revenue under the premise of the market to maintain long-term development has become a crucial problem to solve for major enterprises. Only good pricing strategy can better capture the market and establish a stable customer relationship.

According to the characteristics of product life cycle, the development of cloud services can be divided into five stages: the introduction stage, the stage of development, the rapid development stage, the mature stage, the decline stage. At present, most CCS providers charge fees to customers based on fixed pricing methods. The most commonly used fixed pricing model mainly has three kinds: quantity pricing, book pricing and grade pricing [2]. However, the traditional fixed pricing strategy cannot accurately response the dynamic change of market conditions and the relationship between supply and demand, and also cannot achieve supplier's long-term interests. [3-6] there are a few scholars from the perspective of dynamic inspection of cloud services pricing issues, obviously better than the fixed pricing model, more effectively reflect the changes on the demand of the market. But the current literature of CCS pricing is still inadequate, mostly involving or fixed pricing, lack of the dynamic pricing mechanism of CCS and market strategy research, mostly stay in the short term the lack of long-term interests of thinking.

CCS pricing on whether it can successfully realize commercialization plays a key role. Therefore, this paper chooses CCS pricing mechanism as the object, and hopes to provide useful reference for the future research. Unlike the previous literature reviews [7-9], this paper focuses on the increase of the dynamic study of the review. Put forward practical suggestions for future research. This paper introduces the concept of CCS and the development present situation at first. The next section reviews he related works about three fixed pricing models, and finally pointed out the lack of them and related research suggestions.

\section{The Life Cycle Characteristics of CCS Commercialization}

According to the characteristics of the product life cycle, CCS development has five stages: the introduction stage, the stage of development, the rapid development stage, the mature stage, the decline stage. Different stages have different characteristics. Each stage of technology, investment costs, user acceptance, competition between the market providers, and the role of the price determining will be changed as shown in table 1.

Table 1. The characteristics of CCS at different stages.

\begin{tabular}{|c|c|c|c|c|c|}
\hline Stage & CCS technology & Input cost & Acceptance degree & Market & Price influence \\
\hline introduce & start & a huge sum & low & monopoly & provider \\
\hline development & development & reduce & increase & oligopoly & oligopoly \\
\hline rapid growth & rapid progress & further decrease & rapid increase & imperfect competition & competition + supply and demand \\
\hline mature & mature & minimum & generally accepted & free competition & supply and demand \\
\hline decline & eliminate & abandon & abandon & sign out & \\
\hline
\end{tabular}

CCS is in the introduction period, only individual providers to invest a lot of money. Technology is not mature, and the market acceptance is not high. Sales are very low. With the development of cloud computing, the technology is relatively improved, so the degree of recognition is also increased and sales have increased. The competition among the oligopoly providers began to intensify. With the further development of technology, cloud computing into a period of rapid development. Market recognition and market share is also expanding. The effect of competition between oligopoly providers on pricing has declined; the changes in supply and demand are affected by the increase in the price of the foot. The last is the product into the mature stage, the market gradually into a fully competitive environment. At this point the impact of a single supplier for the price is minimal, because this time the price depends on the supply and demand relationship. Business costs have also been reduced to a minimum, the highest level of technology to reach. Major suppliers are only the price of the recipient, in the rapid development of cloud services today, the service is still not considered a recession, the fifth wave of the IT sector needs to be done. 


\section{The Present Situation of the Cloud Service Pricing}

At present, the CCS is in a rapid development stage. Domestic and foreign study of CCS pricing is at the exploration stage. Research results mostly are fixed pricing. The author of [10] according to whether the price change and depend on the characters of the pricing form is divided into pay-as-you-go, price discrimination and dynamic pricing: Fixed pricing, including pay as you go and price discrimination, do not change according to the customer characters, also does not have dependencies, and not based on real-time market conditions; Dynamic pricing is based on the time, the supply and demand change, asset value, customer value change factors such as.

\subsection{Fixed Pricing}

Because the principle is simple, consumers are easy to understand and accept. So the major cloud service providers generally use the fixed pricing. Establishing the model and solving, analyzed the copyright pricing and pay-as-you-go two models of the optimal pricing strategy and found pay-as-you-go can effectively cover the entire consumer groups [11].Wu etc. through the study of different types of consumers to provider's optimization problem, analysis usually order pricing in order to gain more profit than pay-as-you-go [12]. Now, CCS offers mainly adopts two tiered pricing and reserve pricing form [13].

CCS providers need technical support and high-tech talent, as well as a large data center to run before the operation. Such as Amazon global have 28 databases, about 2,000,000 servers in the background as a cloud carrier. Therefore, the provider needs a large amount of money in the early stage, and most of them are sunk costs. As the user pays relatively less rent, the recovery period is relatively long. So only have the strength of the company to enter the industry, it is easy to form a development period of the high threshold phenomenon. The oligopoly has a strong impact on pricing. The work of [14]-[15] simplified the competition between the major providers into monopolistic competition, oligopoly market. It is concluded that under the cooperative game, the monopoly firm has no strong influence on pricing, and the interests of the two companies and the market share of the dual oligopoly market are symmetrical. But the research is based on the simple monopoly and the assumption of the double oligopoly.

In the period of CCS into the import, most of the suppliers choose the pay-as-you-go mode. At this point, the cost and resources are calculated to provide the feasibility of the use of paid mode. With the development of cloud computing, the two stage pricing can be better suited to the customers with long-term needs. But fixed pricing only from the perspective of the supplier pricing, the lack of customer utility.

\subsection{Price Discrimination}

After start-up stage, price discrimination is better choice. Mainly according to the characters of services, customer characters, quantity and the difference of the value of the customer to pricing. Service characters depending on the pricing mechanism, for example, in this model of CCS might use ladder pricing method, each layer providing the calculation of fixed specifications (such as storage, memory allocation, CPU type and rate).

The authors in [16] introduced the theory of bilateral market, from the angle of two phase charge model, aiming at the condition of the user single ownership and part of the ownership model. It is concluded that when using two-stage charge, CCS providers should take the way of low charge registration fees and high transaction costs, and price discrimination helps to CCS providers to improve their competitiveness. When the user is more belonging, providers can improve user registration fees, reducing the developers to grab market share. According to the principle of price discrimination in economics to work out the price of the different groups, the largest supplier can make consumer utility benefit maximization, achieving win-win situation. This stage, because the cost is still a major factor in the block of investors, so the impact of oligopoly market pricing cannot be ignored.

With the CCS development, due to the diversification of the product, and the increased demand of customers, the use of a simple price will lead to the difficult of allocation of resources. The supplier should consider the rationality of the price in many dimensions. At the same time ensure the interests of consumers when maximize their own profits. This will not lose the market share, but also consolidate their position in the market. At present, cloud service providers are using fixed pricing and price discrimination, ignoring the supply and demand changes in the market. Dynamic pricing strategies can be adjusted and optimized based on time, quality of service and demand and other factors, so that both the provider and the user benefit.

\subsection{Dynamic Pricing}

Dynamic market pricing: under the competitive environment, product price will not change because of the individual; the final price is determined by supply and demand relationship. Fixed pricing and price discrimination, which are adopted by the CCS providers, ignore the changes in the market supply and demand. Dynamic pricing strategy can be based on factors such as time, quality of service and demand change under the condition of adjustment and optimization, so as to benefit both providers and users. The work of [17] proposed the theory of price elasticity of the goal to maximize demand and profit, by means of indirectly control the market demand, establishing a mathematical model to solve the optimal pricing interval. Now, the dynamic pricing based on utility has become the consensus of scholars. Marian compared to fix through the experiment, dynamic comparison under two kinds of ways. It is concluded that dynamic pricing can achieve a better allocation of resources, increase the social overall economic benefit [18]. Sewook think CCS providers adopt dynamic pricing, cloud by the low price strategy to encourage users who are not sensitive to the time to migrate 
the task to the CCS system at low load, which will be beneficial to the load balancing of the providers [19].

Yeo in-depth studying the time, requirements, and resource which impact on pricing, putting forward the automation of pricing mechanism, in order to solve the user demand for services and requirements [20]. The work in [21] established revenue model of providers according to usage, using heuristic algorithm to build the dynamic pricing mechanism under the CCS provider's revenue model. But the model only from the angle of the provider establishing revenue model, without considering the user's interests. Wu put forward the customer perceived value oriented pricing strategy, and implement network evaluation service to the customer product information extract and real-time dynamic mining, provides a way to change a new train of thought and research method of the fixed pricing [22]. But the research results have not been verified by numerical analysis. Guo puts forward the service level agreement (SLA) and return on investment (ROI) in combination with dynamic pricing model to ensure that the provider and consumer interests [23].

In addition, also have the author according to the characters of CCS different utility computing, other dynamic pricing models is putted forward. Compared with the fixed pricing model, the dynamic pricing model can considerably improve the provider's gains. Hu proposed a CCS environment such as a dynamic resource pricing model based on combined double auction (CDA), and gives corresponding algorithm and the simulation experiments. Experiments prove that the resource utilization, users, and the performance of the resource provider's interests better than the fixed pricing algorithm [5]. For example, part of Amazon EC2 is provided according to the spot pricing. Is a good way to improve resource utilization ratio.

Wang regarded CCS resources as a kind of right to use, trading model analyzed by using the theory of option pricing.
Using the option pricing model to establish an improved pricing model of the three forked tree algorithm, and obtained the various factors needed to consider in the pricing process [24]. Yuan through the analysis of the economic characteristics of the SSC, identify the important five factors of pricing. Then these five factors are mapped to the BSM model of option pricing. At last, using the hypothesis test data to simulate, working out the price range of CCS [25]. Although Wang and Yuan all put forward an option pricing model as the prototype of dynamic pricing, but the existing studies are mainly on the interests of the suppliers, and only consider the short-term benefits, the lack of long-term returns.

At present, quite a number of scholars believe that with the rapid development of CCS industry, in order to further seize market stable clients, CCS industry magnates are bound to make further adjustments on the price strategy, such as domestic Ali cloud of CCS is the sixth time this year to lower prices. After the growth period of rapidly rising demand, the product entered the mature period, the market demand tends to be saturated. But at this stage, the cost of enterprise has dropped to the lowest, and reached the highest technical level. At this point, the impact of a single supplier for the price is minimal, because the price depends on the supply and demand relationship.

\subsection{Summary of Findings}

With the vigorous development of the CCS, related research are also gradually increase, while scholars from different angles and methods to study the pricing of CCS, but searches of business model and pricing method are still insufficient and have limitations. The development of each article studies did not identify the specific background, lack of the research on the developing characters in different stages of CCS. The main features and future research recommendations are as follows:

Table 2. The main provider of cloud computing services pricing phase.

\begin{tabular}{llll}
\hline Provider & Production & Service & Pricing \\
\hline \multirow{3}{*}{ AWS } & EC2 m3.medium & Calculation, storage, database & Per hour \\
& S3 & Storage & Pay-as-you-go \\
Google & spot instance & Calculation, storage, infrastructure & Spot instances \\
& App Engine & Infrastructure, web & Pay-as-you-go \\
Ali Cloud & OSS & storage & Pay-as-you-go \\
& ECS & Web, storage, & Per month, quarter, quarter \\
& UHos \& EIP & Beijing BJP 8G & $486 \mathrm{RMB} /$ month \\
Azure & UDB & Guangzhou BJP 8G & $457 \mathrm{RMB} /$ month \\
& UFile & database & Per month \\
Racks-pace & SSD & storage & Daily Downloads \\
\hline
\end{tabular}

(1) Mostly at a fixed price as the research object, dynamic pricing still need further perfect. As shown in the table 2, a majority of the current providers are still dominated by a fixed price, only the Amazon's idle resources in accordance with the method of spot examples for sale. Dynamic pricing is based on the theory, but the research on CCS dynamic pricing is still not enough. Dynamic pricing is the main trend of the future research.
(2) Take into account the interests of the supplier more than the user's interests. The existing dynamic pricing research still ignores the user's benefit. Future research is not only to consider the level of consumer service level agreement, the cost of utility, but also the customer satisfaction of the service response time, broadband queuing priority and other aspects of the quality of service.

(3) According to the above research at home and abroad can 
be found that most of the studies are still a discussion of the short-term benefits of the provider. With the continuous development of the SSC market, which has experienced the stage of monopoly and oligopoly competition, the final CCS market will develop into multistage competition. Affected by time, demand, contract maturity and the use of satisfaction and other factors, users in the use of a CCS a certain period of time after, will reconsider the choice of other providers. SSC providers need to reformulate pricing strategies to achieve the purpose of seizing market share and gain long-term benefits. CCS providers are faced with a long game process. Therefore, the future research can refer to the game theory to carry on the research of the long-term competition. Such as the theory oligopoly market, incomplete market, Nash equilibrium and so on.

(4) Most of the existing research is theoretical research. Many researches are based the discussion of pricing mechanism or proposing a model. Related simulation experiments are also carried out in the experimental environment, the lack of verification of the actual market data.

\section{The Conclusion and Prospect}

This paper firstly introduces the concept of CCS and General classification of pricing. Then analysis and study past researches according to the characteristics of the product life cycle. Finally points out the shortage of the existing research and the future research direction. Propose the practical reference for the pricing of CCS suppliers.

The domestic and foreign scholars have made a great contribution in this field. Now many scholars are aware of the shortcomings of fixed pricing. Nevertheless, the existing dynamic research lacks the consideration of the user's utility. In addition, it is generally based on the hypothetical experimental data, rather than the actual market data to verify the results. With development of CCS industry, dynamic pricing has become the main trend of future research. The early stage of the CCS providers need to a lot of money, technology, high-tech talent, so only powerful company can well this industry, easily forming the phenomenon "high threshold" in the period. The supplier has a strong influence on the price, so the future research should take the competition between firms into consideration. Maybe, we can use the game theory to carry on the thorough research. The future research should consider long-term interests such as market share. Dynamic pricing mechanism, of course, need a good market environment, needs the government to do a good job in guiding, perfecting the corresponding laws and regulations.

\section{References}

[1] The Ministry of Industry and Information Technology Institute. Sadie Cloud Computing Development white Paper (2015). (2015-04)

http://max.book118.com/html/2015/1104/28492940.shtm

[2] Khajeh-Hosseini A, Greenwood D, Somerville I. Cloud
Migration: A case Study of Migrating an Enterprise it System to Iaas[C]. 2010 IEEE 3rd International Conference on Cloud Computing, Miami, Florida, USA, 2010: 450-457.

[3] Mihailescu M, Teo Y M. Dynamic Resource Pricing on Federated Clouds [C]. 2010 10th IEEE/ACM International Conference on Cluster, Cloud and Grid Computing, Melbourne, Victoria, Australia, 2010: 513-517.

[4] Wang Q, Ren K, Meng X. When Cloud meets eBay: Towards Effective Pricing for Cloud Computing [C], EEEE INFOCOM 2012, Orlando, Florida, USA, 2012: 936-944.

[5] Hu Zhigang, liu yan. Cloud Environment Dynamic Resource Pricing based on Combined Double Auction [J]. Computer engineering, 2012, 38 (8): 19-21.

[6] Wang Bo. Based on the Equilibrium Transport Resources Pricing Strategy Research [D]. Kunming: Yunnan University, 2011.

[7] Lin Xiaowei, Zheng Lijuan. Research on Pricing Mechanism of Cloud Services [J]. Pricing Theory and Practice, 2014, (08): $110-112$.

[8] Chen Jiangtao, Zhang Jinlong, Zhang Yajun. Cloud Computing IT Service Pricing Research Review and Prospects based on Prospect [J]. Science and Technology Management Research, 2014, (10): 154-158.

[9] Yu Hui, Liu Zhengwei, Liu Junpeng, Guo Feng. Research on the Accounting Model of Cloud Computing [J]. Information Technology and Standardization, 2014, (03): 32-35+38.

[10] Samuel. Cloud Computing Services Business Model of Pricing Mechanism Study [J]. Journal of Guangdong Communication Technology, 2012, 05: 11-15.

[11] Dou Yifan. "Cloud Computing" Model, the Optimal Pricing Strategy Analysis Software [A]. China Optimization Program Evaluation and Review Technique and Economic Mathematics Research Institute, Chinese Academy of Sciences Institute of Science and Technology Policy and Management Science, Chinese Journal of Management Sciences, Newsroom. The 13th China Management Science Academic Essays, 2011-6.

[12] Wu Lianggang, Xiao Yongbo. Two Common Cloud Computing Service Pricing Mechanism of Contrast Research [J]. Computer Application Research, 2016, (01): 122-125.

[13] Li Chunlan, Deng Zhonghua, Zhang Wenping. Cloud Service Pricing Strategy Analysis [J]. Journal of Library and Intelligence, 2013. (01): 36-41.

[14] Li X, Gu B, Zhang C, et al. Price Competition in a Duopoly IaaS Cloud Market[C]// Asia-Pacific Network Operations and Management Symposium. 2014: 1-4.

[15] Wei L, Yang X. Revenue Sharing Contract in a Cloud Computing Service Supply Chain with a Monopoly AIP and Multiple Competing asps under asymmetric Information[C]// International Conference on Service Systems and Service Management. IEEE, 2015.

[16] Zhang Rui, Song Xiangling, Tang Bingyong. Cloud Computing Service Pricing Strategy based on Two-phase Charge Study [J]. Journal of Natural Science of Heilongjiang University, 2013, 11: $157-163$.

[17] Chen Donglin, Chen Ling. Cloud IaaS Spot Instance Pricing Method Research [J]. Computer Engineering and Design, 2013, $10,3366-3370$. 
[18] Marian Mihalescu, Yong Meng Teo. On Economic and Computational-efficient Resource Pricing in Large Distributed systems[C]//10th IEEE/ACM International Conference on Cluster, Cloud and Grid Computing, 2010: 838-843

[19] Sewook Wee. Debunking Real-time Pricing in Cloud Computing[C]//11th IEEE/ACM International Symposium on Cluster, Cloud and gird Computing, 2011: 585-590

[20] Yeo C S, Venugopal S, Chu X, et al. Autonomic Metered Pricing for a Utility Computing Service [J]. Future Generation Computer Systems, 2010, 26(8): 1368-1380.

[21] Li Yue, Wang Nianxin, Ge Shilun. Cloud IaaS Service Pricing Mechanism Simulation [J]. Computer and Modern, 2014, (11): 20 to 26 .
[22] Wu Lihua, Zhang Yu. Cloud Computing Services based on Customer Perceived Value Dynamic Pricing Strategy Optimization Method [J]. Computer and Digital Engineering, 2013, (41): 244-246.

[23] Guo Wan, Zhang Xiao. An IaaS Dynamic Pricing Model Based on Combining SLA and ROI [J]. Journal of Computer Research and Development, 2014, (S1): 86-90.

[24] Wang D, Wang Y, Liu J, et al. Pricing Reserved and On-Demand Schemes of Cloud Computing based on Option Pricing Model[C]// Network Operations and Management Symposium (APNOMS), 2013 15th Asia-Pacific. 2013: 1-3.

[25] Yuan Zhekai, Ge S, Wang N. IaaS Cloud Computing Services Pricing based on BSM Model [J]. Computer Application Research, 2014, (11): 3344-3348 + 3356 . 